\title{
DISPOSITIVOS DE FORMACIÓN DOCENTE: EL CASO DE LA TUTORÍA ENTRE PARES
}

\author{
Carolina Clerici ${ }^{*}$ \\ Instituto de Profesorado Sedes Sapientiae, Argentina \\ clericicarolina@hotmail.com \\ Liliana Silvina Lucca ** \\ Instituto de Profesorado Sedes Sapientiae, Argentina \\ lisilucca@gmail.com \\ Rocío Eliana Naef ${ }^{\text {** }}$ \\ Instituto de Profesorado Sedes Sapientiae, Argentina \\ rocionaef@gmail.com
}

Recibido: 10/06/2020 - Aceptado: 24/11/2020

\section{Resumen}

Este artículo expone avances de una investigación en curso que buscó evaluar la Tutoría Entre Pares (TEP) como dispositivo y estrategia de formación docente utilizados en la unidad curricular Práctica Docente del Profesorado de Inglés de un Instituto de Formación Docente terciario no universitario de Gualeguaychú, Argentina. Se desarrolló un estudio exploratorio-descriptivo desde una lógica inductiva de generación conceptual, mediante el diseño de teoría fundamentada. Se realizaron entrevistas a los estudiantes de primer año participantes de la TEP. En este artículo se incluyen categorías como: animarse a preguntar, equilibrio entre lo personal y lo académico, comparación entre tutores y experiencia del propio tutor como factor facilitador u obstaculizador de la acción tutorial. La participación en la TEP, como oportunidad de trabajo entre pares, implica un rol activo para el tutor y el tutorado en cuanto al proceso de aprendizaje, en aspectos académicos y, además, en cuestiones vinculares, de relación con otros y con la institución en la que estudian. El análisis de la información empírica da cuenta del valor de la TEP como dispositivo y estrategia de formación docente. Resta un camino por recorrer para conocer la voz de tutores, coordinadores y docentes de asignaturas de primer año.

Palabras clave: Tutoría entre pares - Rol del tutor - Formación docente - Dispositivo - Estrategia.

\footnotetext{
* Magister en Procesos Educativos mediados por Tecnologías por la Universidad Nacional de Córdoba. Licenciada en Educación por la Universidad Nacional de Quilmes. Profesora de Inglés y portugués. Profesora regular de Metodología de la Investigación en la Universidad Nacional de Entre Ríos. Profesora de Práctica Docente en el Instituto de Profesorado Sedes Sapientiae de Gualeguaychú, Entre Ríos.

** Profesora de Enseñanza Superior en Filosofía y Pedagogía por Universidad de Concepción del Uruguay. Especialización en Formación de Formadores. Diplomatura en Didáctica Universitaria por la Universidad de Concepción del Uruguay.

**k Licenciada en Lengua Inglesa. Profesora de Inglés. Profesora del curso de ingreso al Profesorado de Inglés en el Instituto de Profesorado Sedes Sapientiae de Gualeguaychú, Entre Ríos.
} 


\title{
TEACHER EDUCATION DEVICE: THE CASE OF PEER TUTORING
}

\begin{abstract}
This article presents findings of an ongoing research that sought to evaluate Peer Tutoring (PT) as a device and strategy for teacher training used in the subject Teaching Practice of English Teachers in a Tertiary Non-university Teacher Training Institute in Gualeguaychú, Argentina. An exploratory-descriptive study was developed through the inductive design of grounded theory. Interviews were conducted with the first year students participating in the PT. This article includes categories such as: encouragement to ask, balance between the personal and the academic, comparison between tutors and the tutor's own experience as a facilitating or hindering factor of the tutoring action. Participation in the PT, as an opportunity for peer-to-peer work, implies an active role for the tutor and the tutors in terms of the learning process, both in academic aspects and as regards their relationship to others and to the institution in which they study. The analysis of empirical information shows the value of PT as a device and strategy for teacher training. Further research needs to be carried out in order to know the voice of tutors, coordinators and teachers of first-year subjects.
\end{abstract}

Keywords: Peer tutoring - Role of the tutor - Non-university teacher training - Device - Strategy.

\section{Introducción}

En este artículo se exponen avances de una investigación en curso que buscó evaluar la Tutoría Entre Pares (TEP) como uno de los dispositivos de formación utilizados en la unidad curricular Práctica Docente del Profesorado de Inglés de un Instituto de Formación Docente terciario no universitario de la ciudad de Gualeguaychú, Argentina. En Entre Ríos en 2014 y 2015 se implementaron los nuevos Diseños Curriculares en los Institutos de Formación Docente; en aquellos la Práctica Docente se entiende como un dispositivo, por ello resulta pertinente aclarar el alcance de este concepto (CGE, 2014). Según Souto et. al. (1999) un dispositivo es "un espacio potencial que da lugar a lo nuevo, al cambio, al desarrollo de la educabilidad del sujeto, de la grupalidad y de lo instituyente" (Souto, 1999, p. 38). Para Sassi y Yasbitzky (2008) los dispositivos son espacios, instrumentos o engranajes que se crean y se utilizan para resolver problemáticas ajustadas al contexto. En este sentido, un dispositivo de formación profesional se orienta intencionalmente a la producción y transformación pedagógica del sujeto a través de la experiencia de formación. Por lo tanto considerar la Práctica Docente como un dispositivo implica encararla con un criterio de innovación, en tanto esta significa novedad y cambio, puesto que lo nuevo adquiere un sentido diferente que incluye maneras nuevas de hacer algo ya realizado. De ahí que Práctica Docente, en los nuevos diseños, adquiera no solo otras significaciones sino también nuevos modos de realización y articulaciones diferentes. Específicamente el Diseño Curricular del Profesorado de Inglés, en referencia al Campo de la Formación en la Práctica Profesional, establece que este campo posibilita abrir espacios de aprendizaje que permiten recorridos pedagógicos flexibles en la formación de los estudiantes. Además, este mismo Diseño Curricular en relación con las intervenciones de los estudiantes en la Práctica y residencia propone diferentes acciones, entre ellas la intervención en otros roles como bibliotecario/a, preceptor/a o tutor/a.

El trabajo de investigación cuyos resultados se describen aquí pretendió aportar conocimiento sistemático sobre la experiencia de TEP antes mencionada, sobre todo teniendo en cuenta que la búsqueda de antecedentes de investigación de experiencias de TEP en Institutos de Formación Docente de Argentina no arroja prácticamente resultados excepto unos pocos casos que se exponen a continuación.

Prado y Rosetti (2017) se propusieron conocer las características de la implementación de la tutoría en los Institutos de Formación Docente (IFD) de gestión estatal de la Ciudad de Buenos Aires. El trabajo persiguió como objetivos conocer las características, alcances y tensiones de dicha implementación; identificar quiénes son los profesionales que cumplen la función de tutoría y cómo ejercen el rol; analizar las diferentes perspectivas que construyen el sentido de esta función, entendiendo que las tutorías son un recurso para el fortalecimiento de las trayectorias formativas de los estudiantes. El interés por esta temática surge a raíz de la importancia que cobra el dispositivo de las tutorías en la educación superior en la Ciudad de Buenos Aires, como política educativa de acompañamiento a las mencionadas trayectorias formativas. El universo abarca la totalidad de los IFD (21) de gestión estatal de la Ciudad de Buenos Aires, que incluye a las Escuelas Normales Superiores (ENS); Institutos de Enseñanza Superior (IES) e Institutos Superiores de Profesorado (ISP).

Este informe comienza con la presentación de un enfoque conceptual de la tutoría en los institutos de educación superior y continúa con el análisis de la información a partir de tres ejes: la tutoría desde una perspectiva política, la tutoría desde una perspectiva institucional y la tutoría desde una perspectiva pedagógica. En este estudio se da cuenta que hay escasa producción académica referida al tema para este subsistema, ya que la mayoría de los estudios y/o investigaciones están enfocados a experiencias llevadas a cabo en Universidades y no en los Institutos de Formación Docente.

La Dirección General de Educación Superior de la Ciudad de Buenos Aires (2018) reúne las narraciones acerca de las experiencias sobre la intervención tutorial desarrolladas durante 2016 en diversas 
instituciones de nivel superior de la ciudad de Buenos Aires. Si bien en este escenario se apunta a la figura del profesor tutor como quien acompaña, apoya y asesora a los estudiantes en su proceso de integración, desarrollo y formación pedagógica ayudándolos a superar las dificultades que se les presentan a lo largo del proceso educativo, aparece también la figura del tutor par. La función tutorial de los docentes se orienta al acompañamiento de los procesos de aprendizaje de los alumnos en proceso de formación, en los que se requiere orientación para la organización y metodologías de estudio, inserción grupal, lectura y escritura académica, preparación para exámenes, competencias vinculares, etc.

Por otra parte, aparece también un proyecto de "Pares tutores" destinado a alumnos avanzados a quienes se les ofrece un proceso formativo para el acompañamiento que brindarán a los alumnos ingresantes. Por ello se recupera, para este apartado, el trabajo de Álvarez (2018) que expone la experiencia realizada en el IES N ${ }^{\circ} 1$ "Dra. Alicia Moreau de Justo". Se trata del "Taller de lectura y escritura académica con un enfoque creativo" que fue dictado por estudiantes del profesorado de Lengua y Literatura, del instituto mencionado. El taller estuvo destinado a estudiantes de primer año de la carrera de Psicopedagogía, sin restricción en su acceso para aquellos alumnos de los profesorados que desearan asistir. Los tutores pares acompañaron a los ingresantes en el proceso de alfabetización académica y en los modos de leer y escribir específicos que supone la misma

En cuanto a resultados de la experiencia llevada a cabo la autora expresa en los comentarios de cierre que los resultados alcanzados indican que los estudiantes estiman valiosa la función tutorial por el acompañamiento y cuidado que brinda. Y específicamente la TEP se dimensiona como una experiencia que ofrece la oportunidad de vivenciar un enseñar y aprender entre todos a la vez que promueve el trabajo cooperativo entre estudiantes, entre docentes, y entre estudiantes y docentes.

$\mathrm{Si}$ se intenta efectuar una mirada histórica del tema tutoría con el objetivo de observar su desarrollo, se puede ubicar su origen en la Edad Media, junto con el nacimiento de las universidades. Rodríguez Espinar (2012) afirma que la función tutorial, en tanto acompañamiento de la formación de los alumnos, ha constituido parte consustancial de la tarea docente, desde ese momento, en la historia de la educación. Por su parte Lobato et. al. (2004), en referencia a la figura del tutor, expresan que la misma se instituyó como autoridad encargada de guiar los conocimientos y de acompañar a los alumnos. Las Universidades de origen medieval experimentaron un proceso de transformación a principios del siglo XIX, momento en el cual se puede ubicar su tránsito hacia los que se constituirían como tres modelos clásicos de universidad: el alemán, el francés y el anglosajón. En este último, según López García (2009), la tutoría pasa a ser un elemento esencial de la actividad docente. De acuerdo con este autor, el concepto tutoría abarca realidades diferentes: la tutoría académica y la mentoría. La primera consiste en la guía que un docente ejerce sobre la formación de un alumno. Por otra parte, en cuanto a la segunda, su primer registro histórico queda establecido en la antigüedad, precisamente, en la "Odisea" de Homero. En esta obra se narra que Ulises, antes de emprender su viaje, le encomienda a su amigo Mentor la educación y guía de Telémaco, único hijo de aquel. Por ende, de ahí deriva la denominación mentoría, y esta función central en tanto relación de apoyo de una persona con mayor experiencia, el mentor, hacia otra con menor experiencia, el mentorizado.

Según Valverde Macías et. al. (2004), la mentoría es un:

proceso de feedback continuo de ayuda y orientación entre el mentor (alumno de curso superior que atesora los conocimientos y habilidades necesarios para ayudar), y un estudiante o grupo de estudiantes de nuevo ingreso, con la finalidad de paliar las necesidades de estos y optimizar su desarrollo y potencial de aprendizaje (Macías et. al., 2004, p. 92).

En este trabajo se retoma el concepto de tutoría, en especial como tutoría universitaria y, el mismo se constituye en punto de partida para el análisis de una experiencia de tutoría en el nivel superior no universitario. La implementación de la misma, en la actualidad, responde a diversas problemáticas que se presentan en el nivel superior. Entre ellas se mencionan las dificultades para el ingreso y para el egreso, la deserción y, también, la formación académica insuficiente o poco adecuada para enfrentar los desafíos educacionales propios de este nivel académico. En este sentido cabe consignar que Hanne y Mainardi (2013) aluden al dispositivo tutorial y lo definen como un espacio para apoyar, orientar y acompañar el aprendizaje, además de promover la adaptación al medio universitario. Una de las modalidades de la tutoría universitaria es la TEP.

Esta modalidad de tutoría tiene una larga tradición histórica, ya que fue puesta en práctica como método de enseñanza mutua por el pedagogo Joseph Lancaster en el siglo XIX (Vidal Bueno, 2015). La misma consistía en el desempeño de alumnos aventajados como instructores de sus compañeros con menos conocimientos, lo cual implicaba la actuación del docente como educador indirecto. Cabe aclarar que el método lancasteriano estaba destinado a las escuelas primarias.

La TEP, además de ser considerada un dispositivo como se afirma más arriba, constituye para Villagra-Bravo y Valdebenito-Zambrano (2019) una estrategia para la formación del profesorado. Organizaciones internacionales como la UNESCO (Delors, 1996) y la OCDE (2005), destacan la interacción en grupos diversos como una competencia fundamental para el desarrollo del individuo. En su trabajo las autoras mencionadas establecen una relación entre esta competencia y el aprendizaje entre iguales. "Entendemos que estructurar las interacciones de los individuos a través de métodos de aprendizaje cooperativo - como se presenta en esta investigación - brinda oportunidades de aprendizaje a los estudiantes a través del diálogo, la reflexión y la negociación de significados" (Villagra-Bravo y Valdebenito- 
Zambrano, 2019, p.2) Si bien estas autoras utilizan la denominación Tutoría entre iguales, es coherente recuperar lo que afirman en cuanto a estrategia de formación para aplicarlo a la TEP.

Por otra parte, la tutoría entre iguales como estrategia para la formación favorece el desarrollo de prácticas más inclusivas en el nivel universitario que responden a los diversos desafíos en esta etapa, no solo en términos de aprobación-reprobación o retención-deserción, sino en términos más cualitativos, que consideren los procesos de incorporación desde una perspectiva más personal y social (Villagra-Bravo y Valdebenito-Zambrano, 2019, p.4).

La actividad tutorial en su extenso desarrollo histórico ha ido adoptando diversas formas y significados. En el caso de la TEP, Topping (2005) la define como la adquisición de conocimientos y habilidades a través de la ayuda activa y apoyo de pares o compañeros. También puede definirse como un conjunto de situaciones educativas en que los estudiantes tienen oportunidades recíprocas de aprender y enseñar; de aprender de y con los otros, tanto en situaciones formales como informales. La TEP representa una ocasión para compartir el conocimiento, las ideas y las experiencias entre los participantes. Duran y Vidal (2004), la entienden la como un método de aprendizaje entre iguales basado en la creación de parejas, con una relación asimétrica (derivada del rol de tutor o de tutorado que desempeñan respectivamente), con un objetivo común, compartido y conocido (adquisición de una competencia académica), que se logra a través de un marco de relación planificado por el profesor.

En cuanto a las formas y modalidades, actualmente se pueden mencionar diferentes tipos de tutorías con carácter de asistencia al estudiante. Topping (2001) menciona (peer tutoring), modelado entre pares (peer modeling), observación entre pares (peer monitoring), mentoría entre iguales (peer mentoring), entre muchos otros, los cuales persiguen las mismas finalidades consistentes en brindar ayuda para superar dificultades académicas y ofrecer una forma de acompañamiento al estudiante. En virtud de ello se puede afirmar que las TEP potencian la solidaridad y la cooperación entre ingresantes y estudiantes avanzados. Estos, desde su lugar, pueden favorecer aprendizajes inherentes al contexto de la educación superior y facilitar la inserción de los ingresantes al ámbito académico de la misma. En síntesis, los conceptos de interacción y mediación resultan esenciales en la definición de las TEP en tanto procesos que atraviesan todas sus formas.

Respecto de las ventajas que ofrece el dispositivo TEP, siguiendo a Arbizu et. al. (2005), se pueden agrupar en las específicas para el tutorado, para el tutor y las que se consideran conjuntas. En el primer caso se valora la ayuda personalizada, la orientación para los estudios de nivel superior y para la integración en este nivel académico, inclusive la atención de aspectos más personales pero que inciden en la formación. Desde el punto de vista de los tutores, esta función representa una oportunidad para adquirir y desarrollar competencias cognitivas, sociales y, en cierto modo, profesionales más avanzadas. En cuanto a las ventajas conjuntas, se señala que la TEP promueve el aprendizaje del estudiante que asume el rol de tutor y a la vez, el de su compañero, quien, al ser tutorizado, aprende al recibir la ayuda ajustada que el primero le ofrece. Otro aspecto ventajoso es el contexto de proximidad que se genera y que a la vez facilita, en un clima de confianza, que el tutorado pueda preguntar y expresarse sin temor al error, y ello derive en una reducción del estrés y la ansiedad. Además se ha observado que los alumnos suelen ser mejores mediadores que los propios profesores. Esta afirmación se fundamenta en que los alumnos tutores han sido aprendices, más recientemente. Dicha circunstancia implica que pueden conocer mejor las demandas 0 necesidades de los tutorados. Además, cuentan con la ventaja de compartir referentes culturales y lingüísticos y con ello se genera mayor proximidad socio-afectiva.

Si bien el presente trabajo se centra en conocer la valoración que los tutorados hacen de la tutoría y del tutor, en función de este objetivo es necesario contar con referencias acerca de la figura del tutor par.

En cuanto al perfil del tutor par, se pueden señalar algunos rasgos. Se trata de un alumno regular, que tiene habilidad y disposición para el trabajo colaborativo y que, además, manifiesta capacidad comunicativa y de liderazgo. Otros rasgos destacables y deseables son la tolerancia, el respeto y la empatía. También se puede mencionar que, en la TEP, de algún modo, desaparece la autoridad del tutor, con el rango de la autoridad propia del docente, y esto le permite aparecer como un par y como un aliado para el logro de los objetivos de los tutorados.

En lo que respecta al rol del alumno tutor, se puede afirmar que es un agente coadyuvante y un colaborador estratégico que interviene en el fortalecimiento del desempeño académico de sus compañeros, mediante la orientación y motivación en el proceso de retroalimentación académica y pedagógica.

De acuerdo con Islas y González (2014), es el tutor quien pone al servicio del tutorado todas sus capacidades y cualidades para proveerle información y orientarlo de forma objetiva y desinteresada hacia el logro de sus proyectos académicos y de su autonomía como estudiante.

En una investigación sobre el rol del tutor en general, Capelari (2009) identifica distintas configuraciones del mismo que podrían ser aplicadas al rol del tutor par. La autora extrapola el constructo acuñado por Litwin (1997) quien lo refirió a las estructuras de las clases universitarias y lo aplica al ejercicio del rol del tutor universitario, al que concibe "como una compleja trama de significados y prácticas que se constituyen y desarrollan en los distintos contextos institucionales para responder a problemáticas específicas (Litwin, 1997, p.2)". La autora plantea también que los resultados de esta investigación permiten generalizar interrogantes, a la vez que favorecen la reflexión crítica, en otros contextos, acerca de cómo se configura un rol directamente vinculado con el aprendizaje de los estudiantes. De ahí que se retome su 
análisis ya que el mismo puede orientar el trabajo sobre la TEP en instituciones de nivel superior no universitarias. Las configuraciones que identifica son las siguientes:

Configuración I: "El tutor como parte de un dispositivo institucional de tipo remedial para solucionar dificultades situadas en los alumnos".

Configuración II: "El tutor como orientador que brinda respuestas personalizadas a distintas necesidades y problemáticas de los estudiantes".

Configuración III: "El tutor como orientador/promotor de aprendizajes académicos en los alumnos". Configuración IV: "El tutor como una forma especial de ser docente".

En relación con las actividades que realiza el tutor se pueden mencionar diferentes ámbitos en los que se pueden desarrollar las mismas: académico, de orientación, administrativo, de gestión y de servicios. Las acciones que se pueden desarrollar dentro de estos, tienden a que el tutorado llegue a dimensionar las problemáticas que se le presentan en su vida escolar y tome las decisiones acertadas que le permitan avanzar hacia el éxito académico y, en el futuro, al profesional.

El objetivo principal de la investigación de la que deriva este artículo fue conocer cómo describieron sus experiencias de TEP los estudiantes ingresantes al Profesorado de Inglés en un Instituto de Formación Docente terciario no universitario de la ciudad de Gualeguaychú, Argentina. Además, se plantearon como objetivos específicos indagar la valoración que realizaron los participantes acerca de la experiencia general; averiguar cuáles obstáculos y facilitadores se les presentaron en el proceso; indagar la significación que tuvo para los ingresantes trabajar con estudiantes avanzados; y averiguar en qué aspectos la realización de las TEP ayudó durante el período de ingreso a la educación superior. En este artículo se incluyen las categorías que surgieron con más fuerza de los datos empíricos: la TEP como espacio para animarse a preguntar, equilibrio entre lo personal y lo académico, la comparación entre tutores y valoración que hace el ingresante de la experiencia del tutor como alumno.

\section{Diseño metodológico}

Se realizó una investigación cualitativa, desde una lógica de generación de categorías de análisis para indagar sobre la TEP como dispositivo de formación docente. La información empírica se recolectó en el marco de una experiencia de TEP con alumnos ingresantes al profesorado de inglés de un Instituto de Formación Docente terciario no universitario de la ciudad de Gualeguaychú, Argentina. La unidad de análisis de este estudio es la tutoría como dispositivo y la unidad de observación es el ingresante que participó de la experiencia. Para esta investigación se tomó como muestra a la primera cohorte de ingresantes desde la unidad curricular Práctica Docente I. A diferencia de los sistemas tutoriales analizados en el marco teórico, los tutores que participaron en la experiencia no fueron seleccionados para formar parte del plantel de tutores, sino que realizaron la actividad tutorial como parte de la asignatura Práctica Docente IV.

Para ello se utilizó el diseño de teoría fundamentada (Glaser y Strauss, 1967; Strauss y Corbin, 2002; Taylor y Bogdan, 1987), que responde a una lógica de generación conceptual. Se trata de un modo de investigar que analiza inductivamente los datos empíricos y genera categorías teóricas.

Las principales estrategias de este diseño son el Método Comparativo Constante y el muestreo teórico. Esto implica que a medida que se avanzó en el trabajo de campo se codificaron y analizaron datos simultáneamente y se desarrollaron conceptos a partir de la comparación continua entre la información empírica y la teoría; también implica que se seleccionaron casos o informantes en la medida que agregaron nueva información para la comprensión del fenómeno bajo estudio. Este diseño permite explicar en un nivel conceptual una acción, en este caso, la experiencia de TEP.

Se realizaron entrevistas en profundidad. Las mismas fueron semiestructuradas y flexibles e incluyeron interrogantes que buscaron conocer las experiencias de los participantes; la valoración de la experiencia; la estimación de obstáculos y facilitadores; y la significación general de la experiencia tanto para los ingresantes como para los estudiantes del último año del profesorado. Este tipo de entrevista facilita un diálogo en el que se pone el foco en el entrevistado, para que se pueda expresar libremente y, a la vez, que el entrevistador pueda realizar contrapreguntas en caso de que se requiera profundizar el contenido de la respuesta.

Para el procesamiento de la información empírica se recurrió al método comparativo constante (Glaser y Strauss, 1967), que consiste fundamentalmente en comparar las conceptualizaciones que surgen de los datos empíricos. Para ello se utilizó una matriz de tres columnas: en la primera se registró el evento o incidente, es decir, la transcripción de entrevistas y observaciones; en la segunda columna se incluyeron comentarios del entrevistador y en la tercera columna se fueron registrando las categorías analíticas que surgieron de la codificación abierta. Luego se construyeron memos con las categorías emergentes y se seleccionaron categorías centrales durante la codificación axial. Finalmente se produjo la escritura de la teoría en la codificación selectiva, acompañada de herramientas típicas de análisis cualitativo como son esquemas, mapas conceptuales y matrices.

La validación de los datos se llevó a cabo a través del trabajo en grupo sostenido de los tres integrantes del equipo de investigación, el análisis individual de la información empírica y la triangulación de la mirada de cada uno en instancias colectivas en las que se acordaron las categorías de análisis. 


\section{Resultados y discusión \\ Animarse a preguntar}

Uno de los aspectos más reiterados entre los entrevistados fue la tutoría como un espacio para hacer preguntas que no se animaban a hacer al profesor o frente al resto de sus compañeros. Según Perassi y Doval Temporalli (2014) "las tutorías se basan en la construcción de un vínculo, una situación de comunicación apropiada, un clima de confianza, un espacio donde el o los estudiantes puedan expresar sus inquietudes" (Perassi y Doval Temporalli, 2014, p. 25) En este sentido, los entrevistados expresaron que sentían temor a preguntar en clase, en especial cuestiones asociadas a lo académico y dudas propias de un ingresante.

Para nosotros que recién llegamos nos da vergüenza preguntar ciertas cosas a los profesores o a los adultos (Entrevista 3).

El tutor no llega a ser un profesor, uno no llega a tenerle quizás el miedo que le puede tener a un profesor por hacer una pregunta media tonta, por ejemplo, ¿eh... pero en un parcial qué tengo que hacer? ... dudas mínimas que uno tiene pero que no se anima a preguntárselas al profesor (Entrevista 1).

Poder preguntar, sacarme las dudas que por ahí para preguntárselas a un profesor te daba vergüenza y para alguien que era, no tu par, porque ellos iban a cuarto, pero más o menos parecido, que ya había pasado por lo que vos estabas pasando. (Entrevista 7).

Estos testimonios confirman que la TEP implica un modo diferente de acercarse a la información por tratarse de un espacio que habilita una comunicación que difiere de la de una clase con el docente. Además por el contexto de proximidad que este tipo de tutoría genera, se produce un clima de confianza que ofrece al tutorado la posibilidad de realizar preguntas y de expresarse más libremente que con el profesor, sin temor al error. El hecho de recibir una atención personalizada acorde con sus capacidades y proveniente de un par contribuye a reducir el estrés y la ansiedad que, a su vez, influye en mejoras académicas, mayor motivación y compromiso (Duran Gisbert y Flores, 2014; Melero y Fernández, 1995).

El tamaño de los grupos de la TEP fue percibido de diferente modo por los tutorados. Para algunos, cinco tutorados para cada tutor, como se planteó en esta experiencia, era una cantidad excesiva. Otros destacaron que la tutoría era un espacio de grupos reducidos que facilitaba la atención más personalizada. En ese espacio se generaba más confianza y cercanía que invitaba a preguntar y "sacarse" las dudas. Esto indica que la tutoría es percibida como una ocasión para compartir conocimientos y experiencias, que derivan en un enriquecimiento mutuo. Así la tutoría representó un encuentro apto para potenciar el intercambio, la cooperación y la solidaridad entre los ingresantes y algunos estudiantes avanzados, en línea con Hanne y Mainardi (2013).

\section{Equilibrio entre lo personal y lo académico}

El modo en el que se distribuye el tiempo dedicado a aspectos académicos y a cuestiones personales fue mencionado como uno de los grandes desafíos de la tutoría. Enriquez y Reta (2006) caracterizan a la tutoría como la construcción de una relación pedagógica singular, orientada a la construcción de un vínculo que acompaña al estudiante en su tránsito por el espacio académico. Estas autoras mencionan las dimensiones académica, institucional y socio-relacional en una experiencia de tutoría que realizaron y que resultan posibles de ser observadas en los diferentes grupos de tutorados entrevistados para este trabajo. Para algunos, como ilustra el primer testimonio, predominó el trabajo sobre contenidos; para otros, como se observa en el segundo caso, se dedicó demasiado tiempo a lo personal en detrimento de lo académico.

Era como que ese momento lo queríamos usar más para decir 'equis profesor la verdad que no sé cómo manejarlo, ¿vos qué me recomendás?', cosas más de la vida cotidiana, no tanto de [los contenidos de las materias] (Entrevista 2).

Charlaba mucho y hacía un poquito menos de lo que teníamos que hacer, por ahí, ¿no? Por ejemplo, ella nos pedía que trajéramos una traducción de Fonética y se iba todo el medio módulo hablando de otra cosa y quedaba en el aire [eso que habíamos preparado] (Entrevista 3).

En dos de los casos se logró ese equilibro entre comentarios referidos a lo personal, al funcionamiento de la institución, por un lado, y lo académico, por el otro. Como expresaron:

Pensé que iba a ser más de hablar de las experiencias y eso. Pero al final no resultó tan así, resultó más como un profesor. Pensé que iba a ser más académico, apoyo académico, pero también, si bien fue académico también fue personal (Entrevista 5). 
Ella tiene ese tipo de personalidad que por ahí decía "Bueno, vuelvan al ejercicio", "Presten atención de vuelta", que estaba bueno porque nos ponía ahí, nos llamaba la atención por lo menos, porque éramos varias y nos poníamos a hablar de otras cosas (Entrevista 7).

En este sentido, y en línea con lo que afirman Sola Villazón y De Pauw (2004) la TEP se presentó como una propuesta alternativa complementaria de la enseñanza habitual y, a la vez, como espacio de convivencia donde se habilitó el diálogo entre estudiantes avanzados e ingresantes. En ese diálogo las relaciones de afecto y reconocimiento resultaron tan significativas como la aproximación a los saberes específicos y propios del discurso académico.

\section{Los otros tutores: comparación inevitable}

La comparación entre tutores fue observaba en la mayoría de los casos. Los aspectos en los que fue más notoria dicha comparación fue en cuanto a los modos de vincularse con sus tutorados y también en la forma en que preparaban y desarrollaban el encuentro de tutoría.

En cuanto a las diferencias en el modo en el que se vincularon, algunos manifestaron que, a diferencia de otros compañeros, no lograron con su tutor una relación personal. Expresaron que había quienes se reunían fuera de la institución a tomar mate y tratar los temas de las asignaturas, como queda ilustrado con el primer testimonio. En cambio los dos fragmentos que siguen muestran que no se dio ese tipo de vínculo.

Por ahí no hubo mucha confianza, que hubo con otras chicas, que las otras compañeras tenían otras tutoras que son de acá e iban a la casa, a tomar mate y les explicaban temas (Entrevista 2).

Quizás no era el más pedagógico, había tutores que eran más dedicados en el sentido de que siempre estaban pendientes de sus tutorados pero él era, por su propia personalidad, no había distancia sino que no era común entre nosotros el trato. Nuestro trato no fue más allá de seguirnos en las redes sociales (Entrevista 1).

¡Ella que era tutora de unas compañeras mías, yo decía qué envidia! Cómo se llevan bien con su tutora. Y no era que nuestra tutora era mala, sino que no, no había onda, no sé si no era de mi tipo, capaz era como que no compartíamos las mismas cosas, entonces era complicado eso. Pero no creo que sea ni culpa de ella, ni culpa mía, era algo que no se daba la situación (Entrevista 2).

Otro aspecto que compararon los entrevistados entre sus tutores y los de sus compañeros fue el modo de planificar las tareas propias del encuentro de tutoría. En este caso, reclamaron que otros tutores llevaban actividades y ejercicios para resolver con sus tutorados. En uno de los casos la comparación entre las actividades desarrolladas por distintos tutores- facilitada por el espacio compartido en ese momentoderivó en la decisión de participar de la actividad en otro grupo.

Bueno, yo me voy un ratito con tu tutor o podemos compartir un ratito con tu tutor porque yo quiero también que me expliquen (Entrevista 10).

\section{La propia experiencia del tutor ¿facilitador u obstaculizador?}

En cuanto a la valoración de la experiencia del tutor se puede advertir que la misma es legitimada por provenir de alguien cercano y en situación de paridad como alumno, que ha experimentado recientemente las vivencias propias de quien ingresa a un nivel superior. Diferentes testimonios de los entrevistados dan cuenta de ello:

El tutor está ahí para hablar a partir de su propia experiencia (Entrevista 1).

Tienen los mismos problemas que nosotros, les pasan las mismas cosas que a nosotros, entonces es como que te ayuda mucho y te incentiva (Entrevista 9).

En este sentido es pertinente recuperar lo que afirman Perassi y Doval Temporalli (2014) acerca de los tutores quienes "observan que, al transmitirles a los estudiantes su propia experiencia, muchas veces los tranquilizan y esto les permite seguir adelante con la materia o repensar su estudio" (Perassi y Doval Temporalli, 2014, p.31).

La experiencia de haber tenido que rendir una materia varias veces resultó, para algunos, un beneficio a la hora de tener que explicar los temas. Según Cardozo Ortiz (2011) es más fácil aprender con un igual, pues se tiene mayor facilidad de expresar lo que cada uno conoce y siente. En ese sentido, comentaron:

Fonética se la sabía entera, pobre mi tutora, porque la había tenido que rendir varias veces (Entrevista 7). 
Entonces como que es una guía para, para los chicos de primero, que vos..., ya están desorientados y están en un mundo nuevo, va a entrar, vas a empezar a acostumbrarte a tener una mirada más de un compañero, no tanto de un profesor, porque por ahí un profesor es un poco más distante, pero está bueno para tener a alguien que ya pasó por todas esas experiencias para que te ayude a vivir la tuya también (Entrevista 10).

Si bien reconocieron que la experiencia del tutor resultó valiosa, en algunos casos, mencionaron que no necesariamente sería igual a la propia o bien que no estaban de acuerdo con algunas de sus percepciones y sugerencias acerca de los profesores y lo que acontece en el aula. Como se puede observar en los dos testimonios que siguen:

Nosotros le preguntábamos cómo fue tu experiencia en tal materia. "Ah no, a mí no me gustó porque la profe... qué sé yo, tenés que bancarla..." Bueno, pero igual para qué le preguntábamos nosotros también, después te ponés a pensar como que su experiencia capaz no iba a ser la misma que la mía, y te asustaban (Entrevista 2).

Comentar o decir cosas de profesores no muy positivas, no sé si será una debilidad, pero cuando nosotros íbamos al aula no era así, o no nos parecía lo mismo que ella, entonces por ahí, su punto de vista del profesor, además de lo que es académicamente, nosotros íbamos con una idea previa y cuando llegábamos al aula no era así o no nos parecía eso (Entrevista 10).

Se advierte en los testimonios que el hecho de compartir la experiencia generó mayor confianza entre tutor y tutorados. Como expresaron:

Con estos chicos generás más confianza y aparte en torno de la edad, y de que ellos también son estudiantes, entonces ellos te entienden a vos, que vos recién ingresás (Entrevista 3).

\section{Conclusión}

Esta investigación permitió caracterizar uno de los dispositivos utilizados en la Práctica Docente, específicamente la tutoría entre pares (TEP), en el Profesorado de Inglés de un Instituto de Formación Docente terciario no universitario de la ciudad de Gualeguaychú, Argentina. Puesto que las categorías surgen a partir de un análisis de lo expresado por los tutorados, esta caracterización se construye en la línea de una mirada de la TEP como estrategia que favorece la adaptación a la educación superior, no sólo en términos académicos, sino también a partir de un acompañamiento más personal y social.

Los entrevistados destacaron la TEP como una experiencia valiosa que les permitió encontrar un espacio de confianza en el cual animarse a preguntar. Esto se debe, no solo al tamaño de los grupos, sino también a la cercanía con el tutor como alguien que ha transitado recientemente la experiencia de ingresar a la educación superior. La información obtenida en las entrevistas da cuenta de que este espacio de cercanía generado en la TEP facilitó el animarse a preguntar puesto que liberó a los tutorados de la vergüenza o el miedo que hubiesen sentido al manifestar sus dudas frente a profesores. Esto propició un modo de acercamiento a la información diferente al que se da con frecuencia en el aula.

Otro aspecto de la TEP que fue recurrente en las entrevistas es el equilibrio entre el tiempo dedicado a tratar cuestiones personales y de la vida cotidiana y el destinado a cuestiones académicas, asociadas a contenidos y temas relacionados con las asignaturas. Se percibió con claridad un dilema en este sentido: en aquellos casos en los que predominó uno de estos dos aspectos en detrimento del otro, los tutorados manifestaron la necesidad de una distribución más equilibrada del tiempo dedicado a ambas cuestiones.

Durante las entrevistas también fue posible observar una tendencia a la comparación entre tutores, ya que muchos de los estudiantes describieron su propia experiencia de tutoría en relación con lo que sabían sobre la experiencia de compañeros que habían tenido otros tutores. En la mayoría de los casos se trata de expresiones acerca de cómo les hubiese gustado que fuera su TEP en cuanto a la organización del tiempo y al vínculo con los tutores. Las diferencias que surgieron de la comparación entre tutores estuvieron apoyadas en el hecho de que las tutorías fueron desestructuradas y quedaron a criterio de cada tutor. Teniendo en cuenta que la organización de la tutoría del año siguiente fue unificada, resulta pertinente indagar si se da esa comparación y desde qué criterio se realiza.

Del análisis de la información empírica, la propia experiencia del tutor emergió a la vez como facilitadora y como obstaculizadora de la tarea. En los casos en los que la experiencia servía como orientación para tomar decisiones, fue facilitadora. También lo fue en los casos en los que el tutor se valía de su experiencia para incentivar el estudio, para que sus tutorados no claudicaran ante situaciones adversas propias del ingreso a la educación superior, como puede ser desaprobar un examen o tener que recursar una asignatura. Sin embargo, los entrevistados destacaron que algunos comentarios de los tutores, en relación con docentes y modos de trabajar en clase, no resultaban valiosos cuando lo que observaban ellos mismos no coincidía con lo relatado por los tutores.

En síntesis, la experiencia de participar en la TEP, en tanto oportunidad de trabajo entre pares, implica un rol activo tanto para el tutor como para el tutorado en lo referente a proceso de aprendizaje, en aspectos académicos y, además, en cuestiones vinculares, de relación con otros y con la institución en la que estudian. El análisis de la información empírica da cuenta del valor de la TEP como dispositivo y como 
estrategia de formación docente que intenta atender a ciertas necesidades propias de la educación superior vinculadas con el ingreso, la permanencia y el acompañamiento de los estudiantes de primer año. No obstante, la incidencia del dispositivo en estudio sobre la formación docente requiere una línea futura de investigación que analice en profundidad la voz de los tutores, para quienes el dispositivo fue requisito de la Práctica Docente.

Referencias bibliográficas

Alvarez, B. E. (2018). Taller de Lectura y Escritura Académica: La intervención de los pares tutores con un enfoque creativo. En Dirección General de Educación Superior de la Ciudad de Buenos Aires. La intervención tutorial en la construcción de las trayectorias estudiantiles del Nivel Superior: Narrativas de acompañamiento pedagógico. https://dfdcaba.infd.edu.ar/sitio/revista/upload/la_intervencion_tutorial_en_la_construccion_de_las_trayectorias_estudia ntiles en el nivel_superior.pdf

Arbizu, F.; Lobato, C., y del Castillo, L. (2005). Algunos modelos de abordaje de la tutoría universitaria. Revista de Psicodidáctica 10(1), 7-21.

http://www.redalyc.org/pdf/175/17514745002.pdf

Consejo General de Educación [CGE]. (2014). Diseño Curricular de la Carrera Profesorado de Inglés para la Provincia de Entre Ríos. Consejo General de Educación.

Capelari, M. I. (2009). Las configuraciones del rol del tutor en la universidad argentina: aportes para reflexionar acerca de los significados que se construyen sobre el fracaso educativo en la educación superior. Revista Iberoamericana de Educación, 49(8), 1-10. https://rieoei.org/historico/deloslectores/3110Capelari.pdf

Cardozo-Ortiz, C (2011). Tutoría entre pares como una estrategia pedagógica universitaria. Educación y Educadores, 14(2), pp. 309-325 http://www.scielo.org.co/pdf/eded/v14n2/v14n2a05.pdf

Cieza García, J. (2011). El Proyecto “Tutoría entre Compañeros» en La Facultad De Educación de la Universidad de Salamanca. $\quad$ Aula, $17, \quad$ pp. 249-258 https://gredos.usal.es/bitstream/handle/10366/120864/El_proyecto_\%C2\%ABtutoria_entre_companeros\%C2 \%BB_e.pdf?sequence $=1$ \&is Allowed $=y$

Delors, J. (1996). Learning the Treasure within. Report to UNESCO of the International Commission on Education for the Twenty-First Century. Paris: United Nations Educational, Scientific and Cultural Organization, UNESCO. https://unesdoc.unesco.org/ark:/48223/pf0000109590

Dirección General de Educación Superior de la Ciudad de Buenos Aires. (2018). La intervención tutorial en la construcción de las trayectorias estudiantiles del Nivel Superior: Narrativas de acompañamiento pedagógico.

https://www.buenosaires.gob.ar/sites/gcaba/files/libro tutores con isbn para subircontapas.pdf

Duran, D. y Vidal, V. (2004). Tutoría entre iguales: de la teoría a la práctica. Barcelona: Graó.

Duran Gisbert, D. y Flores Coll, A. (2014). Prácticas de tutoría entre iguales en universidades del Estado español y de Iberoamérica. REICE. Revista Iberoamericana sobre Calidad, Eficacia y Cambio en Educación, 13(1), 5-17. https://revistas.uam.es/index.php/reice/article/view/2796/3011

Enriquez, L. y Reta, L. (2006). La tutoría de pares. Del fast food académico a la construcción de una relación pedagógica singular. En Vera, M. C. y Borja, F. (directores). Reformas universitarias y movimientos estudiantiles en América y Europa. Báez Ediciones, Córdoba.

Glaser, B. y Strauss, A. (1967). The discovery of grounded theory: strategies for qualitative research. Nueva York: Aldine Publishing Company.

Hanne, A. y Mainardi, A. (2013). Reflexiones sobre la inclusión de grupos en situación de vulnerabilidad en la educación superior. El Dispositivo Tutorial: un espacio en construcción. REDU: Revista de Docencia Universitaria, 11(2), pp. 173-192 https://dialnet.unirioja.es/servlet/articulo?codigo=4414189

Islas, D. y González, J. (2014). Tutoría para la calidad de vida y las relaciones humanas. México, D.F.: Umbral.

Litwin, E. (1997). Las configuraciones didácticas. Una nueva agenda para la enseñanza superior. Buenos Aires: Paidós.

Lobato, C.; Arbizu, F., y del Castillo, L. (2004). Las representaciones de la tutoría universitaria en profesores y estudiantes: estudio de un caso. Educación XXI, 7, 135-168. http://www.redalyc.org/articulo.oa?id=70600707

López García, S. (2009). El modelo tutorial en el sistema inglés: análisis de buenas prácticas en los estudios de máster en el London Centre for History of Science, Technology and Medicine del Imperial College of London y el University College of London. [Informe] Universidad de Salamanca. https://gredos.usal.es/handle/10366/75732

Melero, M.; A., y Fernández, P. (1995). La interacción social en contextos educativos. Madrid: Siglo XXI.

Organización para la Cooperación y el Desarrollo Económicos [OCDE] (2005). La definición y selección de competencias clave. Resumen ejecutivo. http://deseco.ch/bfs/deseco/en/index/03/02.parsys.78532.downloadList.94248.DownloadFile.tmp/2005.dscexe cutivesummary.sp.pdf

Perassi, M. y Doval Temporalli, A. (2014). Tutoría entre Pares: fundamentos y descripción de la experiencia en la Facultad de Ingeniería de la UNER. Revista Argentina de Enseñanza de la Ingeniería, 3(6), 23-32.

Prado, S. y Rossetti, M. (2017) Caracterización de los proyectos de tutoría en Formación Docente del sector estatal en la Ciudad de Buenos Aires. Unidad de Evaluación Integral de la Calidad y Equidad Educativa. Ciudad Autónoma de Buenos Aires.

https://www.buenosaires.gob.ar/sites/gcaba/files/ueicee 2017 caracterizacion de los proyectos de tutoria en fd.pdf

Rodríguez Espinar, S. (Coord.) (2012). Manual de Tutoría Universitaria. Recursos para la Acción. Barcelona: Octaedro. https://octaedro.com/appl/botiga/client/img/10162.pdf

Sassi, V. y Yasbitzky, A. (2008, noviembre 26-28) Dispositivos pedagógicos en la formación profesional de los docentes [Ponencia]. I Congreso Metropolitano de Formación Docente, Universidad de Buenos Aires.

Sola Villazón, A. y De Pauw, C. (2004). La tutoría de pares: un espacio para aprender a ejercer el derecho a leer textos académicos. Textos en contexto, 6, 79-94.

Souto, M. et. al. (1999). Grupos y dispositivos de formación. Buenos Aires: Noveduc.

Strauss, A. y Corbin, J. (2002). Bases de la investigación cualitativa (E. Zimmerman trad.). Antioquia: Editorial Universidad de Antioquia.

Taylor, S. y Bogdan, R. (1987). Introducción a los métodos cualitativos en investigación. Barcelona: Paidós.

Topping, K. J. (2001). Peer assisted learning: a practical guide for teachers. Cambridge: Brookline Books. 
ReVista de LA Escuela de Ciencias de LA EduCACIÓN, AÑo 17, NRO. 16, VOL. 2, JULIO A DiCiembRe DE 2021. PÁGINAS 113-122. ISSN 2362-3349 (EN LÍNEA). DISPOSITIVOS DE FORMACIÓN DOCENTE: EL CASO DE LA TUTORÍA ENTRE PARES. CAROLINA CLERICI. LILIANA SILVINA LUCCA. ROCÍO ELIANA NAEF.

Topping, K. J. (2005). Trends in peer learning. Educational Psychology, 25(6), 631-645. https://cmapspublic3.inmc.us/rid=1200100827171_1663553231_5338/Trends_in_Peer_learning_Topping_.pd

Valverde Macías, et. al. (2004) Innovación en la Orientación Universitaria: la Mentoría como Respuesta. Contextos Educativos, 6-7 (2003-2004), pp. 87-112.

https://publicaciones.unirioja.es/ojs/index.php/contextos/article/view/530/494

Vidal Bueno, A. (2015). Aprendizaje cooperativo: tutoría entre iguales como propuesta de mejora de la comprensión lectora [Trabajo Final de Grado]. Universidad Internacional de La Rioja. España. https://reunir.unir.net/bitstream/handle/123456789/3266/VIDAL\%20BUENO\%2C\%20A

Villagra-Bravo, C. y Valdebenito-Zambrano, V. (2019). Tutoría entre iguales como estrategia para la formación del profesorado. Magis, Revista Internacional de Investigación en Educación, 12 (24), 161-176. https://www.researchgate.net/publication/332530107_Tutoria_entre_iguales_como_estrategia_para_la_forma cion_del_profesorado_Peer_Coaching_as_a_Strategy_for_the_Education_of_Teachers 